\title{
Synchronization extension using a bistable galloping oscillator for enhanced power generation from concurrent wind and base vibration
}

\author{
Liya Zhao* \\ School of Mechanical and Mechatronic Engineering, University of Technology Sydney, 15 Broadway Ultimo \\ 2007, NSW, Australia \\ *liya.zhao@uts.edu.au
}

\begin{abstract}
This letter proposes a compact bistable galloping oscillator for achieving enhanced power generation from concurrent wind and base vibration. The harvester consists of a D-shaped bluff body attached to a piezoelectric cantilever, with magnetic interaction introduced between the bluff body and a fixed windward prism. Both theoretical analysis and experiment demonstrate the remarkably broadened synchronization bandwidth for concurrent energy harvesting. In the experiment, the voltage steadily increases from $26.6 \mathrm{~V}$ at $8.5 \mathrm{~Hz}$ to $40.7 \mathrm{~V}$ at $12 \mathrm{~Hz}$, achieving a 10 times wider bandwidth than the linear galloping harvester.
\end{abstract}

The concept of harnessing renewable kinetic energy with low-profile energy harvesters has been enthusiastically pursued in the recent years, aiming to sustainably power ultralow-power electronics like wireless sensors ${ }^{1-9}$. In many circumstances, such as bridges, railway tracks, vehicles, aircrafts, offshore structures, ocean buoys, etc., both wind and base vibratory energy are present. Energy harvesters deployed in such environments are subject to coexisting excitation sources. Since the two kinetic energy sources will unavoidably interact with each other thus influence the overall dynamics and power output, it is inadequate to consider only one type of source in such situations. While previous studies have mainly considered a single type of energy, research on energy harvesting under concurrent excitations has only been conducted very recently with some linear aeroelastic energy harvesters ${ }^{10-14}$.

However, a big challenge with the above linear harvesters is their frequency-dependent susceptibility. Only when the base excitation frequency is very close to the aeroelastic vibration frequency, the latter is locked into the former and the wind and base vibration energy can be truly concurrently harnessed ${ }^{15}$. Although broadband techniques have been widely reported to enhance pure vibration energy harvesting ${ }^{16,17}$, such as nonlinearity, frequency up-conversion, etc., introducing these techniques to the concurrent energy harvesting system will incur complex coupling interactions between the aerodynamic, structural, piezoelectric and electric components, while their effectiveness is not yet clear and must be carefully examined. Due to this challenge, to date, there is very little effort devoted to synchronization widening in concurrent energy harvesting. Very recently, Zhao and Yang ${ }^{15}$ and Zhao ${ }^{18,19}$ theoretically analysed and experimentally validated the superiority of an impact-based energy harvester by installing a mechanical stopper at the bottom of a galloping harvester. Widened bandwidth for effective concurrent energy conversion was achieved. Liu and $\mathrm{Gao}^{20}$ introduced three-to-one internal resonance to a two-degree-of-freedom airfoil-flutter harvester and theoretically showed its power enhancement potential under combined loadings.

This letter proposes a compact broadband galloping energy harvester to achieve substantially enhanced power generation from concurrent wind flows and base vibration. It is confirmed both experimentally and theoretically that introducing bistability is able to widen the synchronization bandwidth for effective concurrent energy harvesting by over 10 times, as compared to what is used in previous literature, i.e., a conventional linear aeroelastic harvester. Moreover, the close-form analytical solution is accurately derived for such a multi-way coupled nonlinear system under dual excitations. What's more, with the wind turbulences, it provides a unique advantage to easily eliminate the difficulty of overcoming the potential barrier that is common to bistable pure vibration energy harvesters. The proposed harvester consists of a D-shaped bluff body attached to the free end of a substrate cantilever, as shown in Fig.1. The flat surface is normal to the incoming wind to give the highest proneness to galloping according to the Den Hartog criterion ${ }^{21}$. In front of the bluff body, a windward prism support is installed. A magnet is attached onto the windward prism and another magnet is embedded in the bluff body. By adjusting the magnet distance, a bistable restoring force will be attained.

The aero-electro-mechanically coupled model for the proposed harvester is given by 


$$
\left\{\begin{array}{l}
M \ddot{\bar{u}}+C \dot{\bar{u}}+K_{0} \bar{u}+F_{n l}+\Theta \bar{V}=F_{\text {air }}-M \ddot{\bar{z}_{0}} \\
\quad=\frac{1}{2} \rho h L \bar{U}^{2}\left[\sum_{i=1}^{3} A_{i}\left(\frac{\dot{\bar{u}}+\dot{\bar{z}_{0}}}{\bar{U}}+\lambda \bar{u}\right)^{i}\right]-M \ddot{\bar{z}_{0}} \\
\bar{I}+C_{p} \dot{\bar{V}}-\Theta \dot{\bar{u}}=0
\end{array}\right.
$$

where $M, C, \Theta$ and $C_{p}$ are the effective mass, damping, electromechanical coupling and piezoelectric capacitance, respectively, $K_{0}$ is the effective stiffness of the initial linear structure in the absence of the windward magnet, $F_{n l}$ is nonlinear restoring force due to the magnetic interaction, $F_{\text {air }}$ is the galloping force, $\bar{z}_{0}=Z \sin \left(\omega_{b} t\right)$ is the base excitation, $\bar{u}$ is the relative bluff body displacement to the base, $\bar{V}$ and $\bar{I}$ are the voltage and current outputs, respectively. In this study, a simple resistor $R$ is considered in the circuit, thus we have $\bar{I}=\bar{V} / R$. The overdot denotes a derivative with respect to the time $t . F_{\text {air }}$ is calculated assuming the quasi-steady aerodynamics ${ }^{22}$, with $\rho, h, L, \lambda$ and $A_{i}$ being the air density, frontal height and length of the bluff body, tip rotation-to-translation ratio and empirical aerodynamic coefficients, respectively. $F_{n l}$ is considered to be a cubic polynomial of the form $F_{n l}=-\alpha \bar{u}+\beta \bar{u}^{3}$ $(\alpha>0, \beta>0)$. If $K_{0}<\alpha$, the system will be bistable. It is worth noting that although Eq. (1) is in the form of a lumped parameter model, it is degraded from and equally effective with a single-mode Euler-Bernoulli distributed parameter model $^{15,23}$
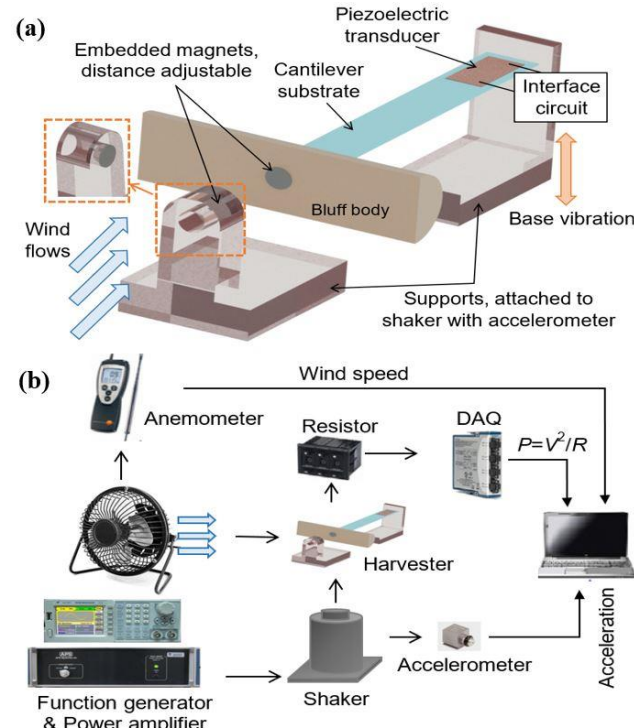

Fig.1 (a) Schematic of the proposed bistable galloping energy harvester for broadband energy harvesting from concurrent wind and base vibration and (b) experimental setup.

A prototype is fabricated with an aluminium cantilever of $135.5 \times 20 \times 0.6 \mathrm{~mm}^{3}$ and a bonded transducer (MFC M2814-P2 from Smart Materials Corp.) of $28 \times 14 \times 0.3 \mathrm{~mm}^{3}$. The D-shaped bluff body is made of polystyrene foam, with $h=32 \mathrm{~mm}$ and $L=107 \mathrm{~mm}$. Including the embedded magnet, the bluff body weighs $4.9 \mathrm{~g}$. The damping ratio is measured using the logarithmic decrement technique. The parameters $M, C$ and $K_{0}$ are calculated based on the equivalent lump parameter transformation method as detailed in our previous studies ${ }^{15,23}$. $\Theta$ is calculated by ${ }^{15} \Theta=$ $\chi / \phi\left(L_{b}\right)=\theta\left(\phi^{\prime}\left(x_{2}\right)-\phi^{\prime}\left(x_{1}\right)\right) / \phi\left(L_{b}\right)$ with $\chi$ being the modal coupling coefficient, $\phi(x)$ being the fundamental mode shape, and $\theta$ being a coupling term expressed by $\theta=-E_{p} d_{31} b_{p} h_{p c} . E_{p}, d_{31}, b_{p}$ and $h_{p c}$ are, respectively, the piezoelectric elastic modulus, piezoelectric constant, transducer width and the distance between the transducer center and the neutral axis of the composite beam cross section. The coordinates $x_{1}, x_{2}$ and $L_{b}$ represent the start and end positions of the transducer and the overall beam length, respectively. The windward prism is made of acrylic glass with a 
magnet embedded in it. The centers of the two magnets are horizontally aligned at rest. If the magnet is removed from the windward prism, the harvester degrades to a conventional linear galloping harvester. The entire experimental setup is illustrated in Fig. 1(b). In the test, a large $R$ of $1 M \Omega$ is employed and the associated voltage is measured with the NI 9229 data acquisition module. The empirical aerodynamic coefficients $\left[A_{1} A_{2} A_{3}\right]$ for the present bluff body are obtained by matching the predicted and measured responses under galloping alone ${ }^{15}$. $A_{1}$ is calculated by $A_{1}=4 \omega_{n} M \zeta / \bar{U}_{c r} \rho h L^{23}$ where $\bar{U}_{c r}$ is the measured short circuit cut-in wind speed, $\omega_{n}$ is the fundamental frequency and $\zeta$ is the measured damping ratio. $A_{2}$ is zero for the present bluff body which is symmetric about the center line in the direction of the incoming flow ${ }^{22,24} . A_{3}$ is determined by matching the predicted voltage levels beyond $\bar{U}_{c r}$ with the measured results. The nonlinear stiffness coefficients $\alpha$ and $\beta$ are obtained by matching the voltage predictions and simulations over a range of frequencies under pure base vibration. The identified properties are summarized in Table I.

\begin{tabular}{cc}
\multicolumn{2}{c}{ TABLE I. System properties } \\
\hline \hline Properties & Cantilever substrate \\
\hline$M(\mathrm{~g})$ & $5.82 \mathrm{~g}$ \\
$\mathrm{C}\left(\mathrm{Nsm}^{-1}\right)$ & 0.0091 \\
$\mathrm{~K}_{0}\left(\mathrm{Nm}^{-1}\right)$ & 35.774 \\
$\Theta\left(\mathrm{NV}^{-1}\right)$ & $8.49 \times 10^{-5}$ \\
$C_{p}(\mathrm{nF})$ & 25.7 \\
{$\left[A_{l} A_{2} A_{3}\right]$} & {$[1.560-6.9]$} \\
$\alpha\left(\mathrm{Nm}^{-1}\right), \beta\left(\mathrm{Nm}^{-3}\right)$ & $57.238,2.096 \times 10^{5}$ \\
\hline \hline
\end{tabular}
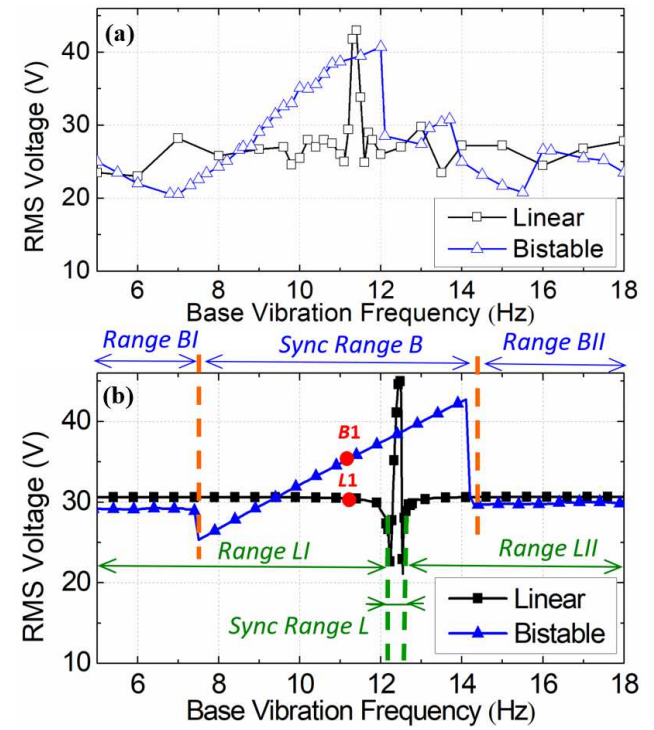

Fig.2 Voltage response from (a) experiment and (b) simulation. The letter ' $L$ ' and ' $B$ ' in the range labels stand for 'linear' and 'bistable', respectively. 


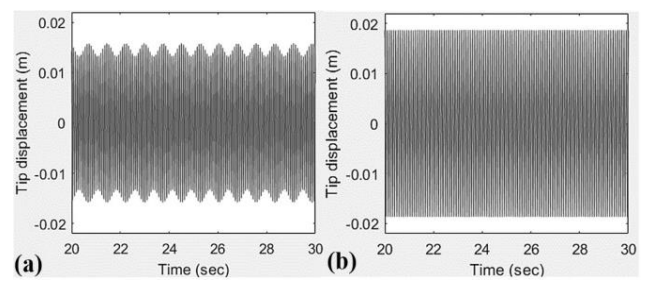

Fig. 3 Time domain displacement response at point (a) $L 1$ and (b) $B 1$.

Figure 2 shows the measured and predicted root-mean-square (RMS) voltage responses as a function of the base vibration frequency $\omega_{b}$. The wind speed is fixed at $4.7 \mathrm{~m} / \mathrm{s}$ and the acceleration is constant at $1 \mathrm{~m} / \mathrm{s}^{2} . \omega_{b}$ is manually swept up. Inspecting the results, both the linear and bistable oscillators' frequency responses can be divided into three ranges (Fig. 2(b)). For the linear harvester, the simulated voltage curve remains generally flat off-resonance in Range LI and Range LII. The voltage level of $30.6 \mathrm{~V}$ in these regions is almost equal to that from galloping alone, meaning that the base vibration energy is not contributing to the power generation. For a random point $L 1$ (Fig.2(b)) at $11.1 \mathrm{~Hz}$, the time domain displacement response is shown in Fig. 3(a). The modulation in the amplitude envelop is caused by the coexisting aerodynamic and base vibratory frequencies. Although the peak deflection is high, the useful RMS displacement is low. When $\omega_{b}$ is very close to the resonance, a voltage peak is observed. In this narrow Sync Range L, the galloping response frequency appears to be synchronized with the base vibration frequency, giving rise to a combined periodic oscillation. In contrast, the bistable galloping oscillator exhibits a significantly broadened synchronization range, i.e., Sync Range B, wherein large-amplitude inter-well oscillations are observed with both wind and base vibration are contributing to energy harvesting. For the purpose of comparison, the displacement profile for point $B 1$ at $11.1 \mathrm{~Hz}$ is shown in Fig. 3(b).

The broadened bandwidth for concurrent power generation is confirmed experimentally in Fig. 2(a). The effective bandwidth is defined here as the frequency range with higher voltage or power output than that from galloping alone. With the bistable galloping oscillator, the voltage steadily increases from $26.6 \mathrm{~V}$ at $8.5 \mathrm{~Hz}$ to $40.7 \mathrm{~V}$ at $12 \mathrm{~Hz}$, achieving a bandwidth of $3.5 \mathrm{~Hz}$ which is over 10 times wider than that of the linear harvester. In general, the simulation and experiment are in good qualitative agreement. The simulation captures the overall trend and well predicts the voltage amplitude within the bandwidth. The voltage amplitude fluctuations can be due to the unstable wind turbulences generated by the fan. The simplified cubic polynomial approximation of the magnetic interaction also accounts for the discrepancies.

To better understand the dynamics of the proposed harvester, a closed-from solution of Eq. (1) is derived using the harmonic balance method ${ }^{25}$. Equation (1) is nondimensionalized as

$$
\left\{\begin{array}{l}
\ddot{u}+2 \zeta \dot{u}+k_{1} u+k_{3} u^{3}+\kappa_{e} v=-\ddot{z}_{0}+\frac{U^{2}}{2 m}\left[\sum_{i=1}^{3} A_{i}\left(\frac{\dot{u}+\dot{z}_{0}}{U}\right)^{i}\right] \\
\frac{v}{r}+\dot{v}-\dot{u}=0
\end{array}\right.
$$

with the dimensionless parameters defined by

$$
\begin{gathered}
u=\frac{\bar{u}}{h}, z_{0}=\frac{\bar{z}_{0}}{h}, v=\frac{C_{p} \bar{V}}{\Theta h}, m=\frac{M}{\rho h^{2} L}, U=\frac{\bar{U}}{\omega_{n} h}, \Omega=\frac{\omega}{\omega_{n}}, \Omega_{b}=\frac{\omega_{b}}{\omega_{n}}, \\
\kappa_{e}=\frac{\Theta^{2}}{C_{p} K_{0}}, r=C_{p} R \omega_{n}, \tau=\omega_{n} t, \omega_{n}=\sqrt{\frac{K_{0}}{M}}, \zeta=\frac{C}{2 M \omega_{n}}, k_{1}=1-\frac{\alpha}{K_{0}}, \\
k_{3}=\frac{\beta h^{2}}{K_{0}}
\end{gathered}
$$

The overdot in Eq. (2) denotes the derivative with respect to $\tau$. For the purpose of generalization, the term in the angle attack that represents the beam tip rotation is ignored. $u$ is considered to be composed of two components, with $u_{g}$ at the galloping oscillation frequency $\Omega$, and $u_{z}$ at the base excitation frequency $\Omega_{b}$, expressed as

$$
u=u_{g}+u_{z}, u_{g}=a_{0} \sin (\Omega \tau), u_{z}=b_{1} \sin \left(\Omega_{b} \tau\right)+b_{2} \cos \left(\Omega_{b} \tau\right)
$$

For $u_{z}$, the focus is on the inter-well oscillations considering that the existence of the disturbing wind turbulences contributes to overcoming the potential barrier as long as the base acceleration is not too small. It is also observed 
in the experiment that the combined oscillations are symmetric once galloping is fully activated. Balancing the terms multiplied by $\sin (\Omega \tau), \cos (\Omega \tau), \sin \left(\Omega_{b} \tau\right)$ and $\cos \left(\Omega_{b} \tau\right)^{19}$ and neglecting any higher harmonics gives the implicit expression for $a_{0}, \Omega$, and $b_{0}=\sqrt{b_{1}^{2}+b_{2}^{2}}$ in Eq. (5). Here, $f_{0}=Z / h$ is the dimensionless base vibration amplitude; and $x_{1}=A_{1} U / 2 m$ and $x_{3}=A_{3} / 2 m U$ are the dimensionless galloping force coefficients. The corresponding voltage amplitudes are given by Eq. (6), while the dimensionless average power is calculated by $p_{a v e}=\left(v_{g 0}{ }^{2}+v_{g 0}{ }^{2}\right) / 2 r$.

$$
\left\{\begin{array}{l}
\left\{\begin{array}{l}
\Omega^{2}+k_{1}+\frac{3}{2} k_{3} b_{0}^{2}+\frac{\kappa_{e} r^{2} \Omega^{2}}{1+r^{2} \Omega^{2}}+\frac{3}{4} k_{3} a_{0}^{2}=0 \\
2 \zeta+\frac{\kappa_{e} r}{1+r^{2} \Omega^{2}}-x_{1}-\frac{3}{2} x_{3} \Omega_{b}^{2} b_{0}^{2}-\frac{3}{4} x_{3} \Omega^{2} a_{0}^{2}=0 \\
{\left[\left(k_{1}+\frac{\kappa_{e} r^{2} \Omega_{b}^{2}}{1+r^{2} \Omega_{b}^{2}}-\Omega_{b}^{2}+\frac{3}{4} k_{3} b_{0}^{2}\right)^{2}+\left(x_{1}-2 \zeta-\frac{\kappa_{e} r}{1+r^{2} \Omega_{b}^{2}}+\frac{3}{4} x_{3} b_{0}^{2} \Omega_{b}^{2}\right)^{2} \Omega_{b}^{2}\right] b_{0}^{2}=f_{0}^{2} \Omega_{b}^{4}}
\end{array}\right. \\
v_{g 0}=\frac{r \Omega}{\sqrt{1+r^{2} \Omega^{2}}} a_{0}, \quad v_{z 0}=\frac{r \Omega_{b}}{\sqrt{1+r^{2} \Omega_{b}^{2}}} b_{0}
\end{array}\right.
$$

Figure 4 compares the analytical solutions with the numerical simulations for different bistability configurations. It is known that the resistance $r$ has a strong influence on the power output. Yet for simplicity, $r$ is fixed at the optimal value for the linear galloping harvester ${ }^{23}$ in all calculations. As a result, the power is optimal for the linear harvester, but smaller than the achievable maximum for the bistable ones. A frequency upsweep is conducted in the simulation since the analysis considers the inter-well response. With the increase of $k_{3}$, the effective bandwidth shifts to the right, and the power peak slightly decreases; while with the increase of $k_{1}$ (absolute value), the bandwidth shifts to the left with a slight increase in the power peak. There is no obvious change in the size of the bandwidth. This bandwidth shifting feature provides a viable solution to adaptively match the ambient base vibration frequency without affecting the extracted wind power. The analytical solutions well predict the effective bandwidth, power amplitude within the bandwidth and the overall trend of power variation. The discrepancy mainly lies in the low-frequency range before entering the power valley. The variations of $a_{0}, b_{0}$ and $\Omega$ are plotted in Fig. 5. It is seen that $b_{0}$ maintains a high level while $a_{0}$ and $\Omega$ remain at zero over the effective bandwidth range, denoting that $u_{g}$ is totally suppressed. As a result, the response is synchronized and periodic, containing only $u_{z}$ at a frequency of $\Omega_{b}$, as reflected in Fig. 3(b). However, it should be noted that this doesn't mean the wind effect diminishes in this range. Inspecting the third equation in Eq. (5), $b_{0}$ is also dependent on the wind speed $U$. In other words, within the bandwidth, both wind and base vibration are contributing to the power generation
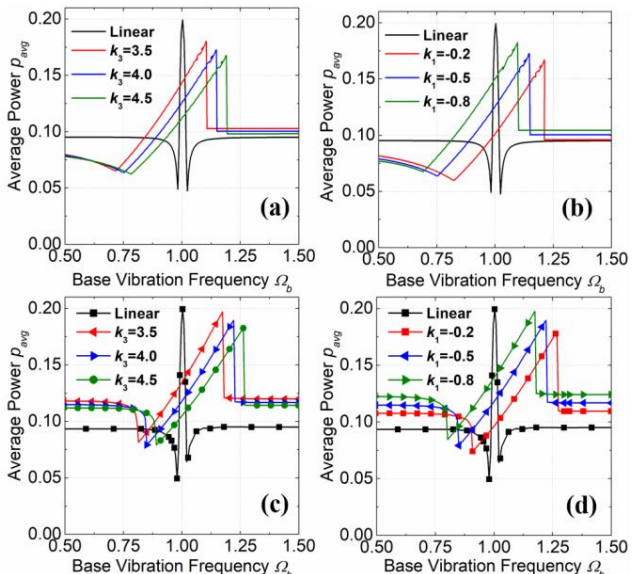

Fig. 4 Power variation with base vibration frequency: (a)(b) analytical solution; (c)(d) numerical simulation. For the bistable curves, $\mathrm{k}_{1}=-0.5$ in (a)(c), $k_{3}=4.0$ in (b)(d). 

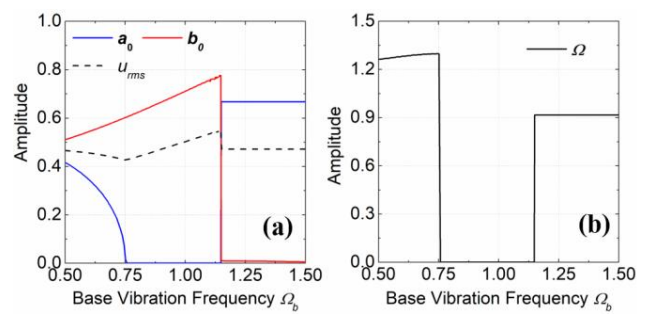

Fig. 5 Frequency responses of (a) $a_{0}$ and $b_{0}$, and (b) $\Omega$. $u_{r m s}$ is the RMS displacement, $u_{r m s}=\sqrt{\left(a_{0}^{2}+b_{0}^{2}\right) / 2}$. Results are obtained with $k_{1}=-0.5$ and $k_{3}=4.0$.

The influences of wind speed and base vibration acceleration on the harvester's performance are depicted in Fig. 6 based on the analytical formulation. It shows in Fig.6(a) that increasing the wind speed results in higher power levels and shifts the effective bandwidth to the right. However, the bandwidth size decreases with increasing wind speed. With increasing base vibration acceleration, it is seen in Figs.6(c) and 6(d) that the bandwidth gets wider and extends to higher frequency ranges.

In summary, this letter proposes a bistable galloping oscillator for enhanced power generation from concurrent wind and base vibration. An aero-electro-mechanically coupled model is developed and experimentally validated. Analytical formulations are also derived. It is confirmed both experimentally and analytically that introducing bistability is an effective means of broadening the synchronization bandwidth in concurrent wind and base vibration energy harvesting.
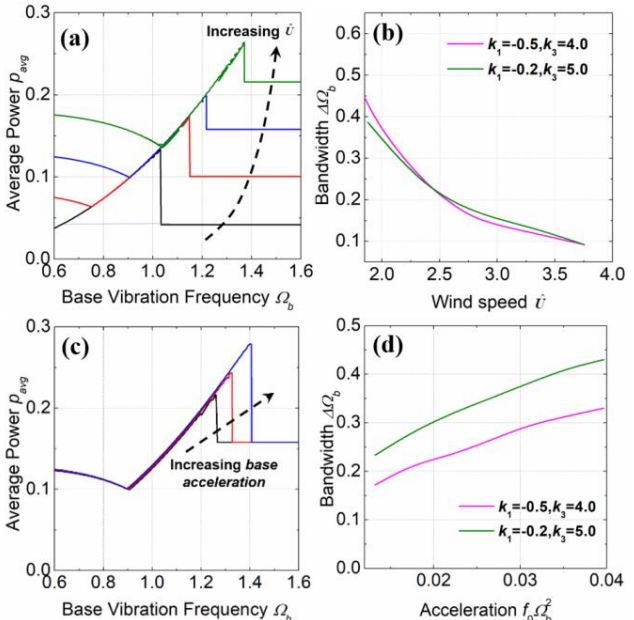

Fig. 6 Variation of (a) power and (b) bandwidth at different wind speeds; variation of (c) power and (d) bandwidth at different base vibration accelerations.

The author would like to acknowledge the financial support from the Blue Sky Grant 2019 of the University of Technology Sydney (321720.2232432).

C. Kitio Kwuimy, G. Litak, M. Borowiec, and C. Nataraj, Appl. Phys. Lett. 100 (2), 024103 (2012).

J. Sirohi and R. Mahadik, J. Vibr. Acoust. 134 (1), 011009 (2012).

L. Zhao and Y. Yang, Smart Mater. Struct. 24 (3), 032001 (2015).

J. A. d. C. Dias, C. De Marqui Jr, and A. Erturk, Appl. Phys. Lett. 102 (4), 044101 (2013).

G. Hu, K.-T. Tse, K. C. Kwok, J. Song, and Y. Lyu, Appl. Phys. Lett. 109 (19), 193902 (2016).

K. Fan, Q. Tan, Y. Zhang, S. Liu, M. Cai, and Y. Zhu, Appl. Phys. Lett. 112 (12), 123901 (2018). 
Y. Shu, W. Wang, and Y. Chang, Smart Mater. Struct. 27 (12), 125006 (2018).

F. Gao, G. Liu, B. L.-H. Chung, H. H.-T. Chan, and W.-H. Liao, Appl. Phys. Lett. 115 (3), 033901 (2019).

S. Zhou, J. Cao, A. Erturk, and J. Lin, Appl. Phys. Lett. 102 (17), 173901 (2013).

P. Chatterjee and M. Bryant, J. Intell. Mater. Syst. Struct. 30 (16), 2405 (2019).

A. Bibo and M. F. Daqaq, Appl. Phys. Lett. 102 (24), 243904 (2013).

A. Bibo and M. F. Daqaq, Smart Mater. Struct. 24 (9), 094006 (2015).

Z. Yan, A. Abdelkefi, and M. R. Hajj, Smart Mater. Struct. 23 (2), 025026 (2014).

H. L. Dai, A. Abdelkefi, and L. Wang, Nonlinear Dyn. 77 (3), 967 (2014).

L. Zhao and Y. Yang, Applied Energy 212, 233 (2018).

R. Harne and K. Wang, Smart Mater. Struct. 22 (2), 023001 (2013).

M. F. Daqaq, R. Masana, A. Erturk, and D. D. Quinn, Appl. Mech. Rev. 66 (4), 040801 (2014).

L. Zhao, proc. of IEEE/ASME AIM, 780 (2018).

L. Zhao, proc. of SPIE 10967, 109671E (2019).

H. Liu and X. Gao, Nonlinear Dyn. 96 (2), 1067 (2019).

J. P. Den Hartog, Mechanical Vibrations. (New York: McGraw-Hill, 1956).

M. P. Païdoussis, S. J. Price, and E. De Langre, Fluid-structure interactions: Cross-flow-induced instabilities. (Cambridge University Press, New York, 2010).

L. Zhao and Y. Yang, Smart Mater. Struct. 24 (7), 075023 (2015),

A. Barrero-Gil, G. Alonso, and A. Sanz-Andres, J. Sound Vib. 329 (14), 2873 (2010).

S. C. Stanton, B. A. Owens, and B. P. Mann, J. Sound Vib. 331 (15), 3617 (2012). 
(a)

\begin{tabular}{c}
$\begin{array}{c}\text { Piezoelectric } \\
\text { transducer }\end{array}$ \\
\hline
\end{tabular}

)

distance adjustable

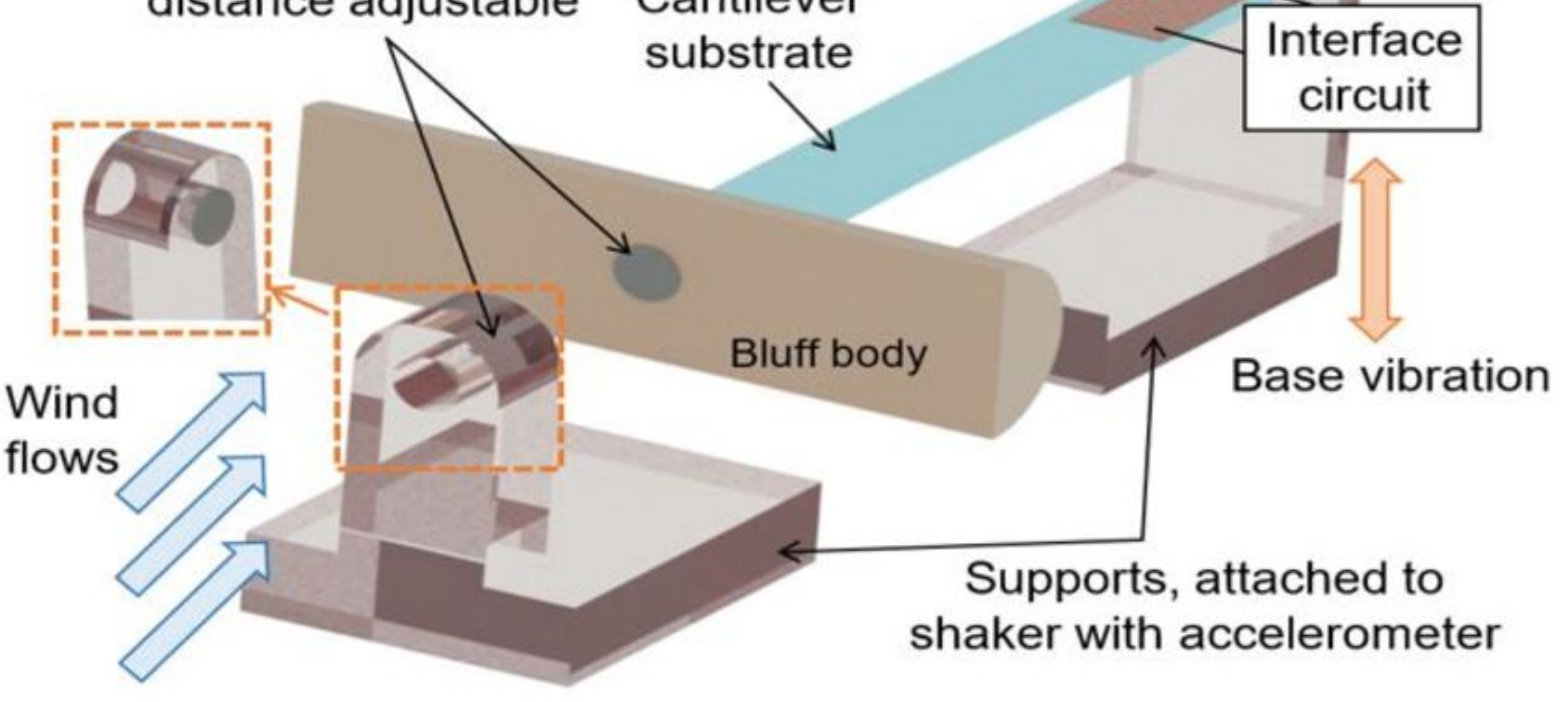

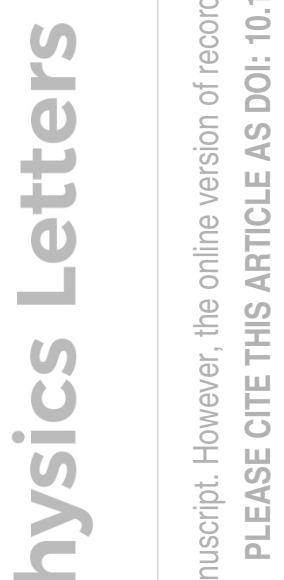

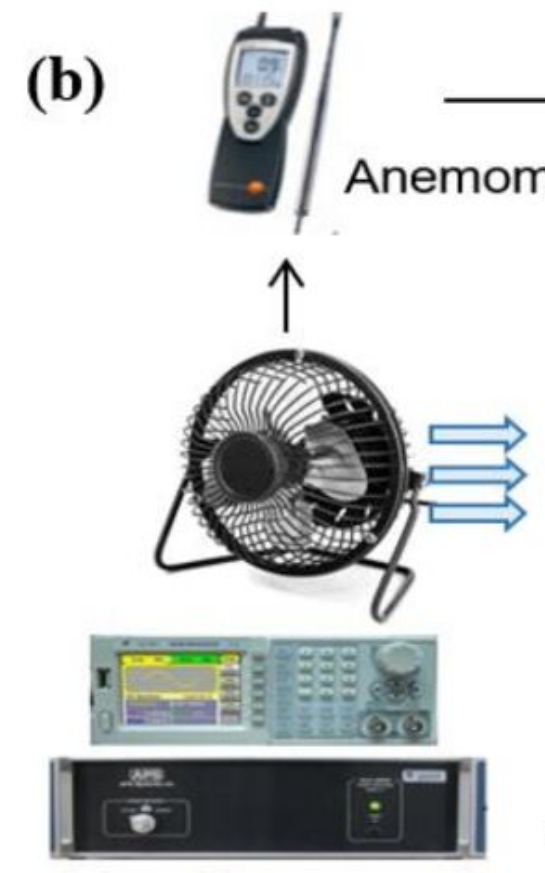

Function generator \& Power amplifier
Wind speed

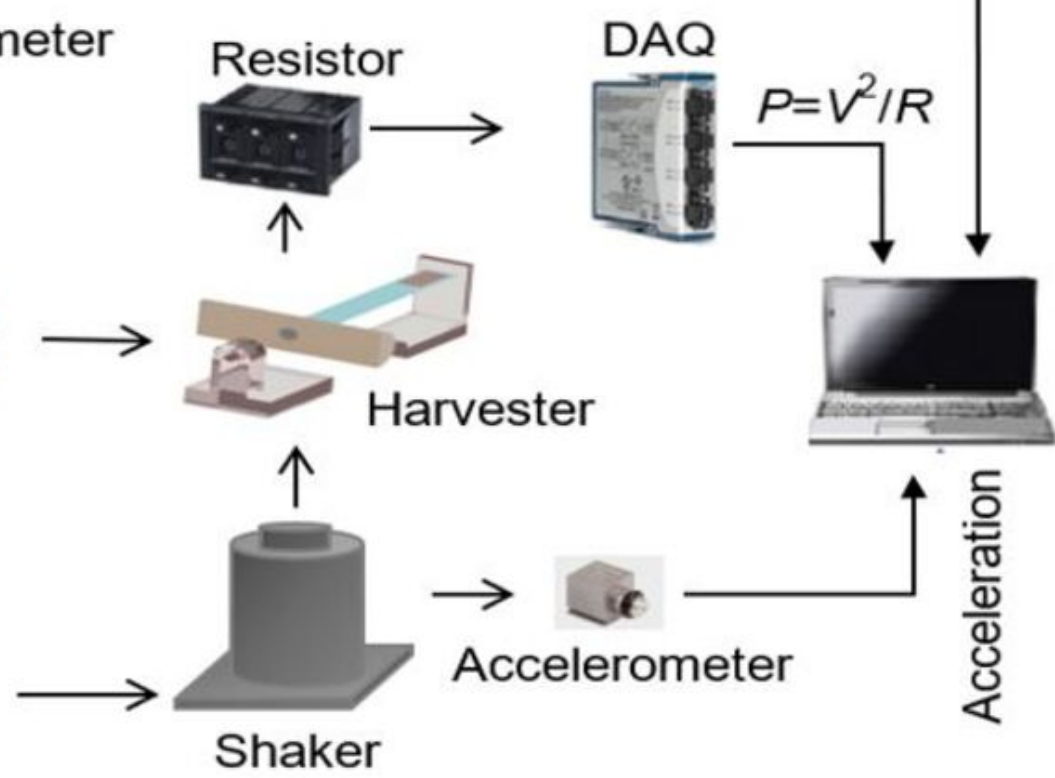



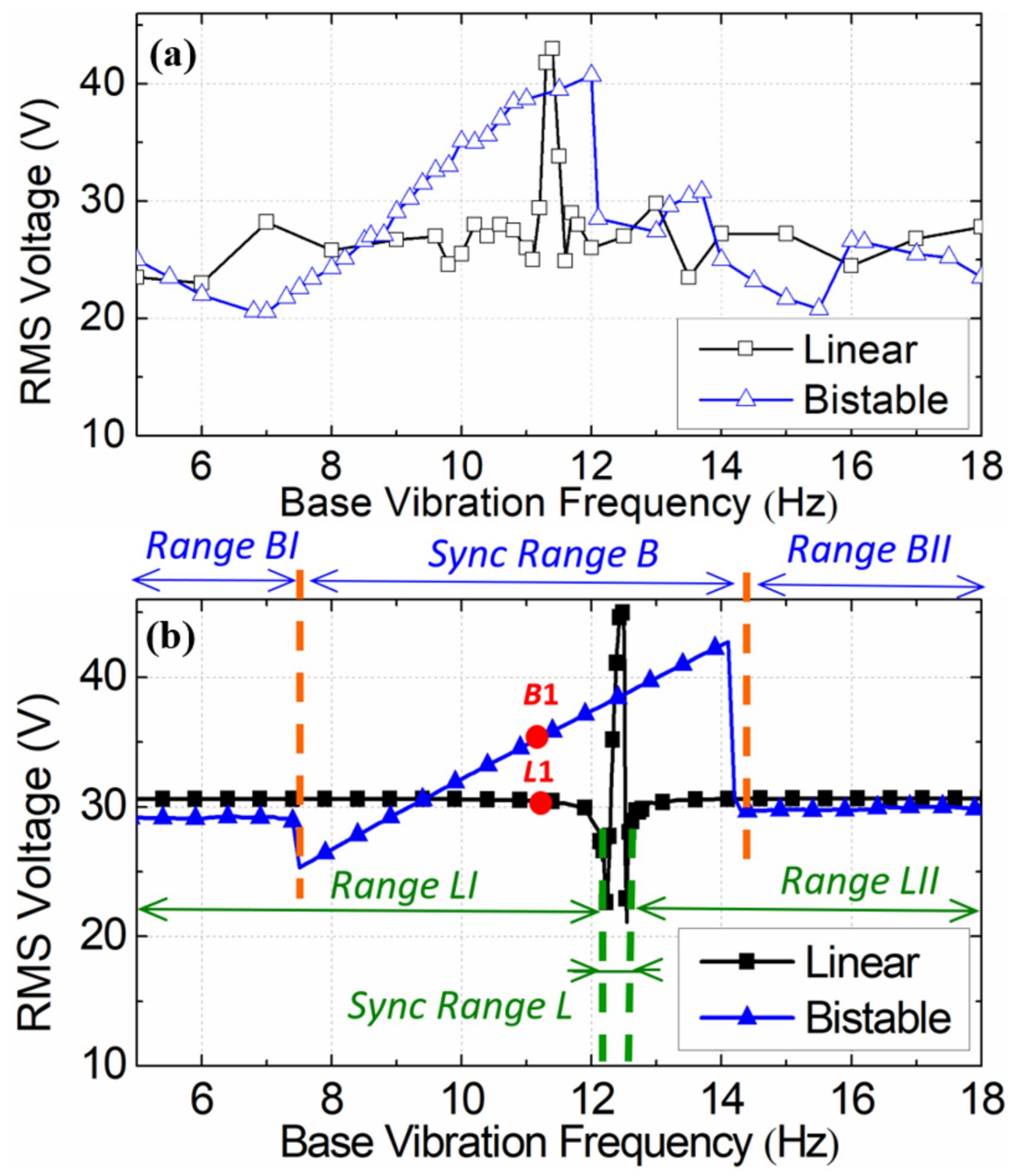


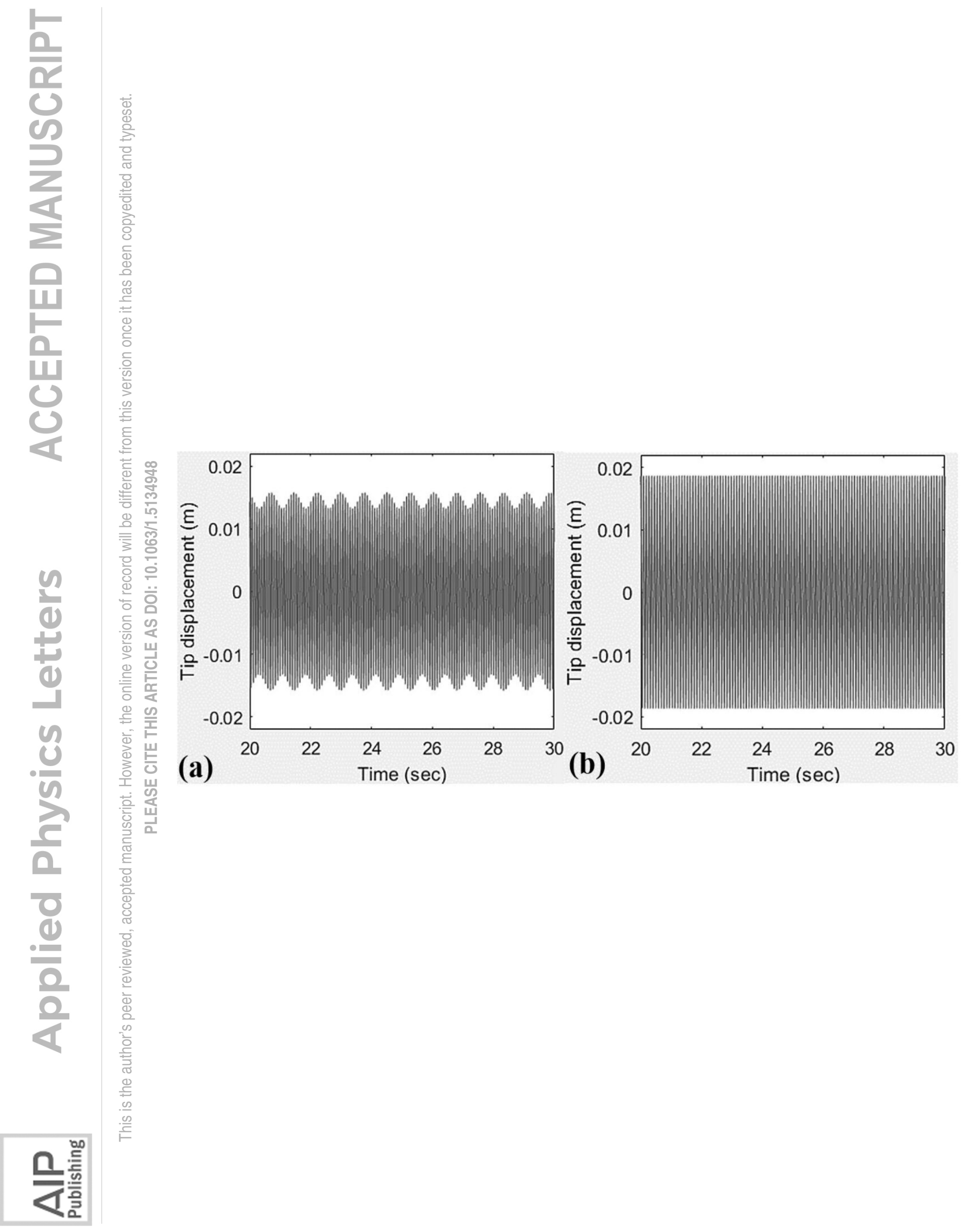



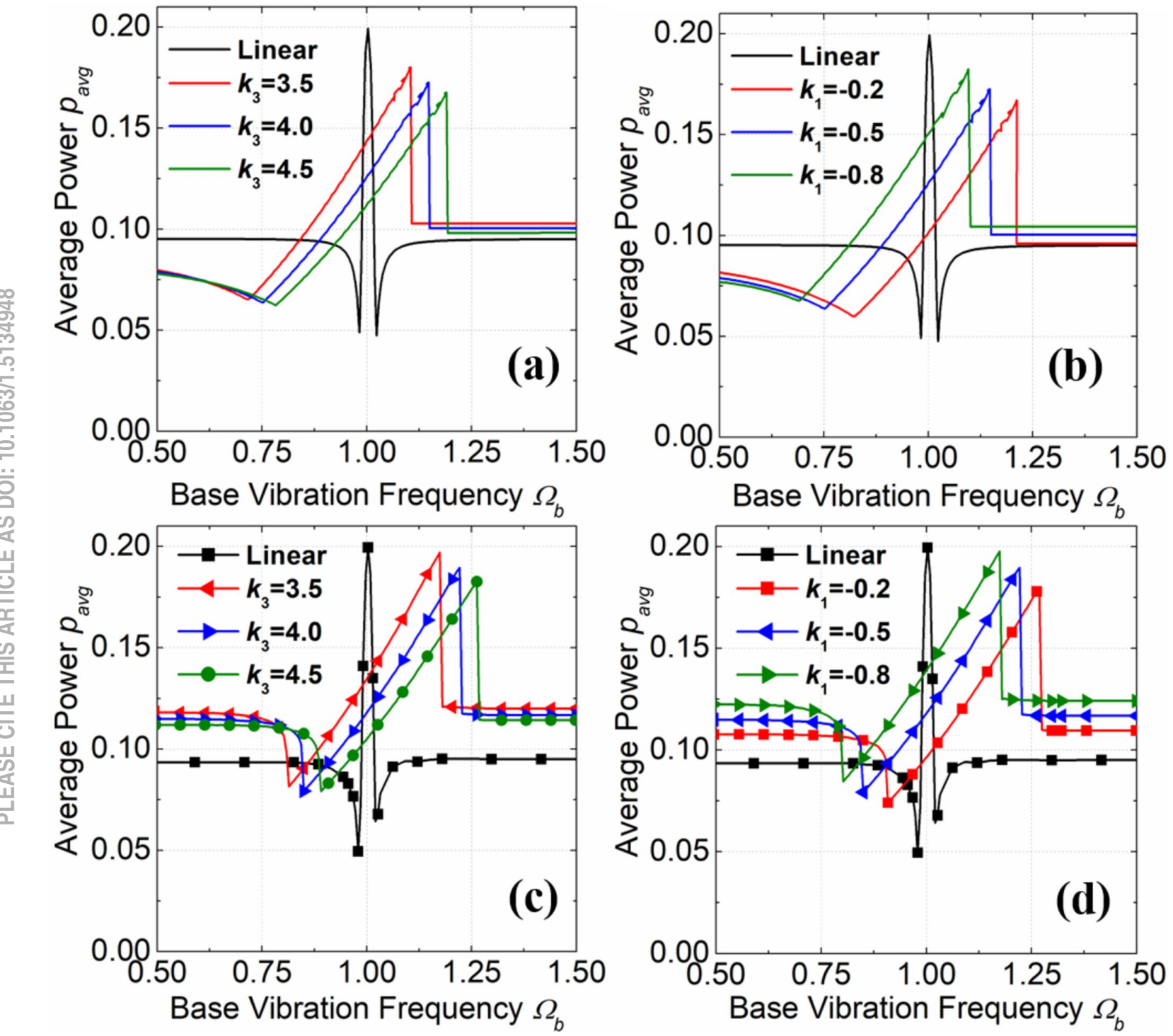

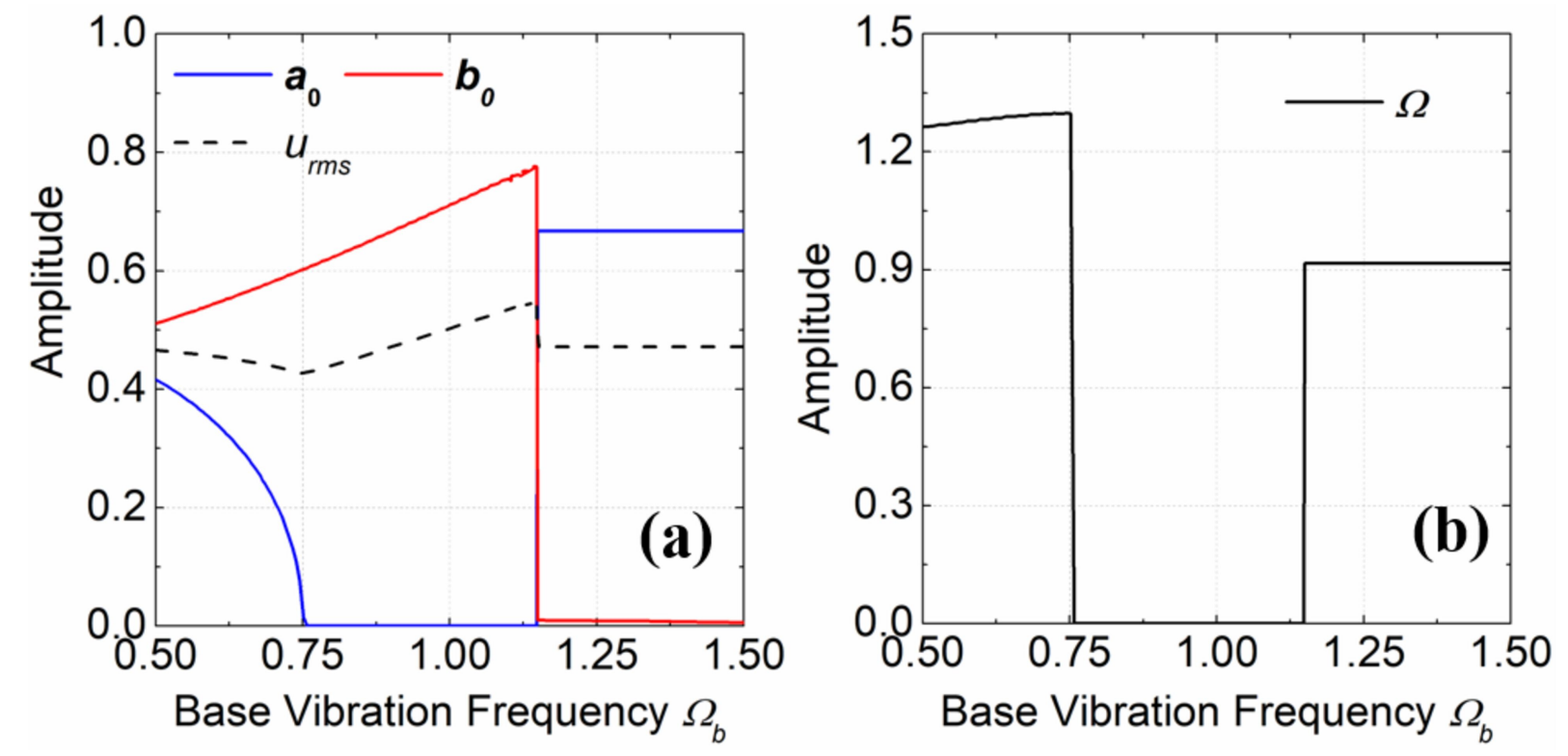

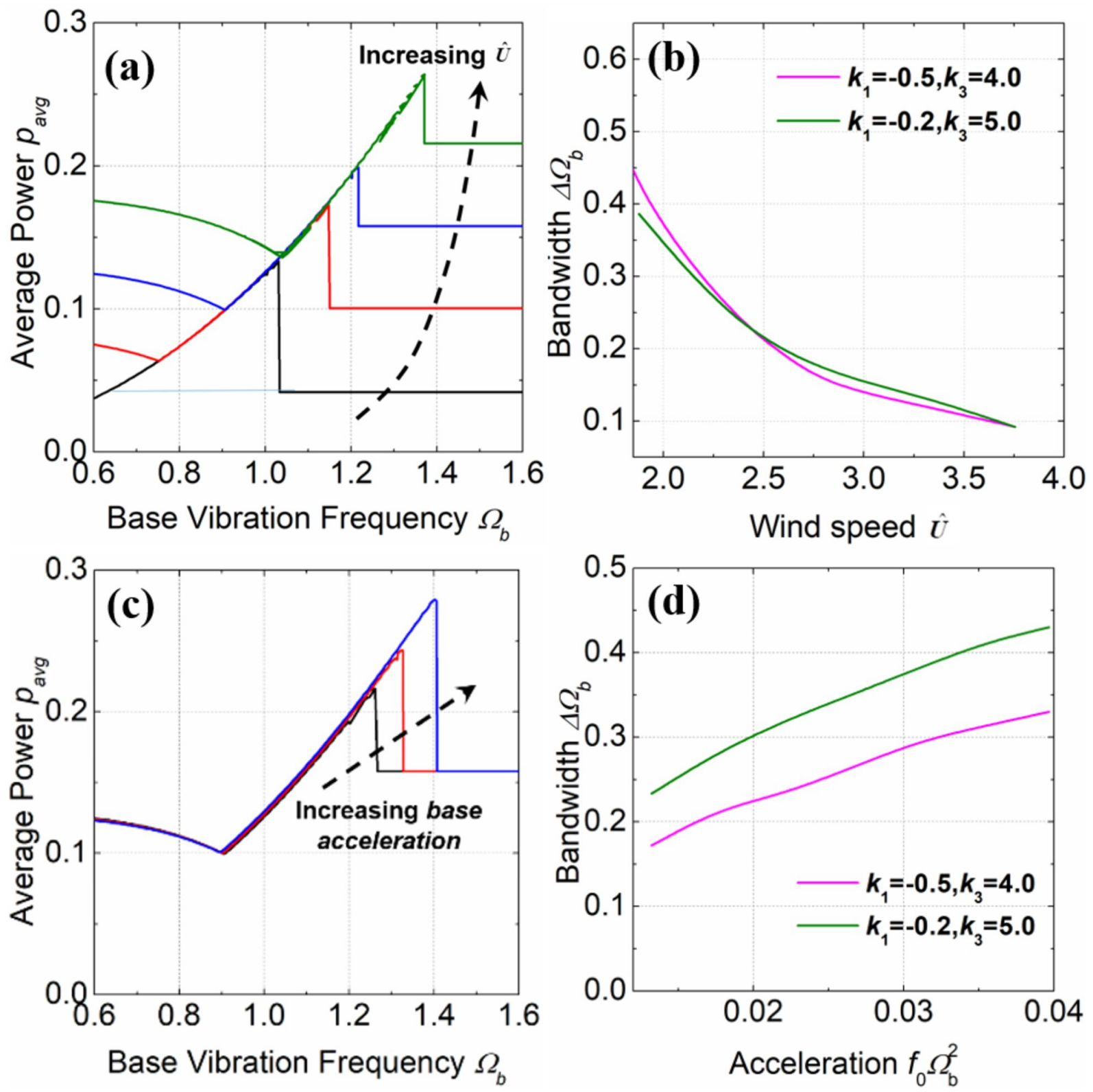